$01,05,11$

\title{
Исследования из первых принципов структурных свойств сплавов Гейслера $\mathrm{Co}-\mathrm{Ni}-\mathrm{Sn}$
}

\author{
(C) А.А. Саносян ${ }^{1}$, В.В. Соколовский ${ }^{1,2}$, О.Н. Мирошкина ${ }^{1,3}$, В.Д. Бучельников ${ }^{1,2}$ \\ 1 Челябинский государственный университет, \\ Челябинск, Россия \\ ${ }^{2}$ Национальный исследовательский технологический университет „МИСиС“, \\ Москва, Россия \\ ${ }^{3}$ Университет Дуйсбурга-Эссена, \\ Дуйсбург, Германия \\ E-mail: arthursanosyan@mail.ru
}

Поступила в Редакцию 8 июля 2021 г.

В окончательной редакции 13 июля 2021 г.

Принята к публикации 16 июля 2021 г.

С помощью первопринципного подхода исследованы структурные и магнитны свойства сплавов Гейслера $\mathrm{Co}_{2} \mathrm{Ni}_{1+x} \mathrm{Sn}_{1-x}(x=0,0.25,0.5,0.75,1)$. Получены зависимости полной энергии кристаллической структуры от степени тетрагональных искажений и выявлено наиболее выгодное кристаллическое упорядочение. Для всех рассматриваемых композиций вычислена энергия магнитокристаллической анизотропии. Показано, что все композиции с обратной гейслеровской структурой обладают анизотропией типа „легкая плоскость“, тогда как в случае регулярной гейслеровской структуры наблюдается смена типа анизотропии с типа „легкая плоскость“ на „легкую ось“ с увеличением содержания Ni. Наибольшая величина анизотропии обнаружена для нестехиометрических композиций $\mathrm{Co}_{2} \mathrm{Ni}_{1.5} \mathrm{Sn}_{0.5}$ и $\mathrm{Co}_{2} \mathrm{Ni}_{1.75} \mathrm{Sn}_{0.25}$.

Ключевые слова: сплавы Гейслера, магнитокристаллическая анизотропия, структурные свойства, устойчивость сплавов.

DOI: 10.21883/FTT.2021.11.51570.12s

\section{1. Введение}

Современный мир невозможно представить без постоянных магнитов на основе магнитотвердых материалов. Они используются при производстве как ставших уже давно привычными аппаратов, так и только появляющихся высокотехнологичных устройств. Примерами могут служить телефоны, наушники, микроволновые устройства, дисковые накопители и другая техника, неотъемлемой частью которой являются постоянные магниты. В производстве магнитотвердые материалы применяются при сортировке отходов, очистке мелкодисперсных составов от металлических примесей, магнитнорезонансных исследованиях и робототехнике. Важное место постоянные магниты занимают также в передовых экологически безопасных технологиях при конструировании электродвигателей и ветряных турбин.

В новейших технологиях наиболее популярными постоянными магнитами являются высокоэнергетические редкоземельные составы на основе $\mathrm{Nd}$, Dy и $\mathrm{Sm}$ [1-7]. Их уникальность объясняется высокими показателями намагниченности, коэрцитивной силы и магнитокристаллической анизотропии (МКА), обусловленными сильным спин-орбитальным взаимодействием. В результате чего несколько граммов такого сплава (например, $\mathrm{Nd}_{2} \mathrm{Fe}_{14} \mathrm{~B}$ ) [1] обладают магнитными характеристиками, сопоставимыми по величине с килограммом карбоновой стали, что позволяет создавать новые миниатюрные высокотехнологичные устройства. Однако высокая стоимость редкоземельных постоянных магнитов препятствует их массовому производству. В связи с этим перед фундаментальной и прикладной наукой стоят задачи поиска и разработки технологии получения высокоэнергетических магнитотвердых материалов, не содержащих редкоземельных элементов. С фундаментальной точки зрения, материал может претендовать на роль постоянного магнита, если он характеризуется высокими значениями спонтанной намагниченности (магнитным моментом), одноосной анизотропии и температуры Кюри. Известно, что магнитные материалы с малой симметрией кристаллической решетки имеют естественный показатель пространственной анизотропии, что дает возможность получить на них большие значения МКА. Можно полагать, что соединения с низкой симметрией кристаллической структуры также могут представлять интерес в производстве постоянных магнитов нового поколения.

Поиски материалов с такими характеристиками активно ведутся среди бинарных соединений $\mathrm{Fe}-\mathrm{Ni}$ [8-10], $\mathrm{Fe}-\mathrm{N}$ [11], $\mathrm{Fe}-\mathrm{B}$ [12], $\mathrm{Co}-\mathrm{Ni}, \mathrm{Mn}-\mathrm{Al}, \mathrm{Mn}-\mathrm{Ga}$ [8], a также трех- и четырехкомпонентных соединений на их основе: $\mathrm{Fe}-\mathrm{Co}-\mathrm{C}[13], \mathrm{Fe}-\mathrm{P}-\mathrm{B}$ с добавкой $\mathrm{Co}$ [14], $\mathrm{Fe}-\mathrm{Si}-\mathrm{B}$ с добавками $\mathrm{P}, \mathrm{S}$, и $\mathrm{Co}[15], \mathrm{Fe}-\mathrm{Co}-\mathrm{B}[12,16]$. $\mathrm{B}$ течение последних десятилетий на роль постоянных магнитов серьезно рассматриваются сплавы Гейслера $X_{2} Y Z$. В ряде работ [17-19], посвященных первопринцип- 
ным исследованиям новых магнитотвердых материалов, рассматривались сплавы Гейслера на основе $\mathrm{Ni}[17,18]$, Fe $[18,19]$, Co $[18,19] \mathrm{Rh}, \mathrm{Au}, \mathrm{Mn}$, а также бинарные составы $\mathrm{Fe}_{3} \mathrm{Ga}$ и $\mathrm{Fe}_{3} \mathrm{Ge}$ [18]. Следует отметить, что в сплавах Гейслера могут наблюдаться тетрагональные искажения кристаллической структуры, вызванные особенностями плотности электронных состояний на уровне Ферми. Таким образом, есть основания полагать, что они должны обладать высокой МКА. В работе [17] для сплавов $\mathrm{Ni}_{2} Y Z$, где $Y=\mathrm{Mn}, \mathrm{Fe}, \mathrm{Co}$ и $Z=\mathrm{B}, \mathrm{Al}$, $\mathrm{Ga}, \mathrm{In}, \mathrm{Si}, \mathrm{Ge}, \mathrm{Sn}$ исследовано влияние элементов $Y$ и $Z$, добавки четвертого элемента, а также деформации решетки на энергию МКА. В работе [19] рассмотрены сплавы $\mathrm{Fe}_{2} Y Z(Y=\mathrm{Ni}, \mathrm{Co}, \mathrm{Pt})$ и $\mathrm{Co}_{2} Y Z(Y=\mathrm{Ni}, \mathrm{Fe}, \mathrm{Pt})$, где $Z=\mathrm{Al}, \mathrm{Ga}, \mathrm{Ge}, \mathrm{In}, \mathrm{Sn}$ для обеих серий. Показано, что для семейств $\mathrm{Co}_{2} \mathrm{NiZ}, \mathrm{Co}_{2} \mathrm{PtZ}$ и $\mathrm{Fe}_{2} \mathrm{PtZ}$, а также стехиометрических композиций $\mathrm{Fe}_{2} \mathrm{NiGe}$ и $\mathrm{Fe}_{2} \mathrm{NiSn}$ c тетрагональной структурой $\mathrm{L}_{0}$ значения МКА лежат в диапазоне от $-12 \mathrm{MJ} / \mathrm{m}^{3}$ для $\mathrm{Co}_{2} \mathrm{PtAl}$ до $+5.19 \mathrm{MJ} / \mathrm{m}^{3}$ для $\mathrm{Fe}_{2} \mathrm{PtGe}$. Для композиций, не содержащих атомов $\mathrm{Pt}$, энергия МКА значительно меньше по величине: от $-2.38 \mathrm{MJ} / \mathrm{m}^{3}$ для $\mathrm{Co}_{2} \mathrm{NiGa}$ до $1.09 \mathrm{MJ} / \mathrm{m}^{3}$ для $\mathrm{Fe}_{2} \mathrm{NiSn}$. В данном случае отрицательный знак говорит о МКА „легкая плоскость“, а положительный - „легкая ось“. Важно отметить, что в большинстве теоретических работ рассматриваются стехиометрические композиции, и мало внимания уделяется исследованиям роли химического и атомного беспорядка в вопросах усиления МКА.

Настоящая работа посвящена исследованию структурных и магнитных свойств сплавов Гейслера $\mathrm{Co}-\mathrm{Ni}-\mathrm{Sn}$ нестехиометрического состава с целью исследования влияния химического беспорядка и атомного упорядочения на МКА. Сообщается, что стехиометрический состав $\mathrm{Co}_{2} \mathrm{NiSn}$ в обратной тетрагональной структуре обладает нулевой МКА [19] и демонстрирует тенденцию к декомпозиции [20] на $\mathrm{Co}, \mathrm{Co}_{3} \mathrm{Sn}_{2}$ и $\mathrm{CoNi}_{2} \mathrm{Sn}_{2}$. В связи с этим интересным представляется рассмотреть возможность стабилизации данного сплава посредством добавки избыточных атомов Со и оценить МКА в получившихся нестехиометрических композициях $\mathrm{Co}_{2} \mathrm{Ni}_{1+x} \mathrm{Sn}_{1-x}$ $(x=0,0.25,0.5,0.75,1)$.

\section{2. Детали вычислений}

Первопринципные расчеты выполнены с помощью программного пакета VASP [21,22] в рамках приближения обобщенного градиента (generalized gradient approximation, GGA), реализованного в функционале Пердью, Бурке и Эрнзерхофа (Perdew, Burke, Ernzerhof, PBE) [23]. Вычисления выполнены на 16-атомной супер-ячейке для двух типов кристаллических структур (рис. 1). Прямая гейслеровская структура L2 1 (пространственная группа $F m-3 m$, № 225, прототип $\mathrm{Cu}_{2} \mathrm{MnAl}$ ) задается следующим атомным упорядочением: атомы Со располагаются в эквивалентных позициях $8 c(1 / 4,1 / 4,1 / 4)$ и $(3 / 4,3 / 4,3 / 4)$, в то время как атомы

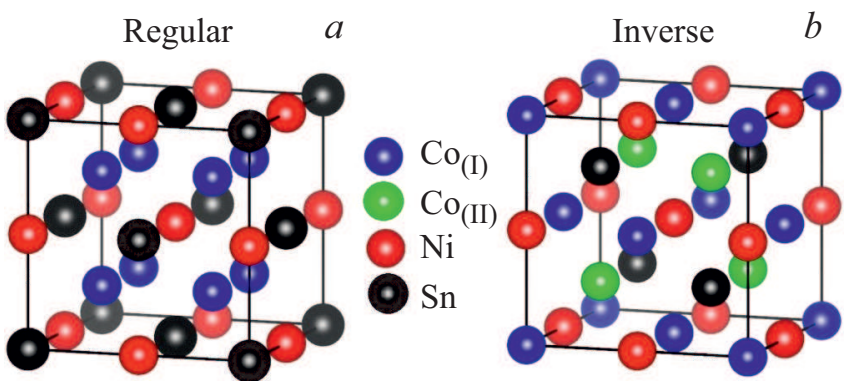

Рис. 1. Прямая и обратная кристаллические структуры сплавов Гейслера $\mathrm{Co}-\mathrm{Ni}-\mathrm{Sn}$.

$\mathrm{Sn}$ и $\mathrm{Ni}$ занимают $4 a(0,0,0)$ и $4 b(1 / 2,1 / 2,1 / 2)$ позиции Вайкоффа, соответственно. Обратная гейслеровская структура (пространственная группа $F-43 m$, № 216, прототип $\mathrm{Hg}_{2} \mathrm{TiCu}$ ) характеризуется расположением атомов Со в неэквивалентных $4 a(0,0,0)$ и $4 c(1 / 4,1 / 4,1 / 4)$ позициях, тогда как Ni и $\mathrm{Sn}$ занимают $4 b(1 / 2,1 / 2,1 / 2)$ и $4 d(3 / 4,3 / 4,3 / 4)$ узлы, соответственно.

Расчеты тетрагональных искажений выполнены при условии равенства объемов аустенитной и мартенситной фаз: $a_{0}^{3}=a_{t}^{2} c$. Здесь $a_{0}$ и $a_{t}, c-$ равновесные параметры кубической и тетрагональной решеток, соответственно. Величина кинетической энергии обрезания составляет $700 \mathrm{eV}$, а кинетической энергии обрезания плоских волн - $500 \mathrm{eV}$. Выбор точек обратной решетки $(k$ точек) осуществлен автоматически при помощи метода Монкхорста-Пака на сетке $16 \times 16 \times 16$.

Энергия магнитокристаллической анизотропии (magnetocrystalline anisotropy energy, MAE) определена следующим образом: $\mathrm{MAE}=E_{100}-E_{001}$, где $E_{100}$ и $E_{001}-$ полные энергии сплавов для случаев ориентации магнитных моментов атомов по направлениям [001] и [100], соответственно. Отрицательный знак МАЕ указывает на МКА вдоль легкой плоскости, а положительный вдоль легкой оси. Расчеты МАЕ реализованы в два этапа. Изначально выполнены самосогласованные расчеты для структуры с коллинеарным упорядочением спиновых магнитных моментов. Затем исследовано неколлинеарное магнитное упорядочение с учетом спин-орбитального взаимодействия. В данном случае расчеты выполнены для ориентаций спинов вдоль [001] и [100].

\section{3. Результаты и обсуждение}

Зависимости полной энергии сплавов $\mathrm{Co}_{2} \mathrm{Ni}_{1+x} \mathrm{Sn}_{1-x}$ $(x=0,0.25,0.5,0.75,1)$ от степени тетрагональности $c / a$ для прямой и обратной гейслеровских структур представлены на рис. 2. Для всех композиций кривые $E(c / a)$ не демонстрируют минимума при $c / a=1$, свидетельствуя об энергетической невыгодности кубической структуры. Данный факт подтверждается расчетами в рамках полной геометрической оптимизации (ионной 

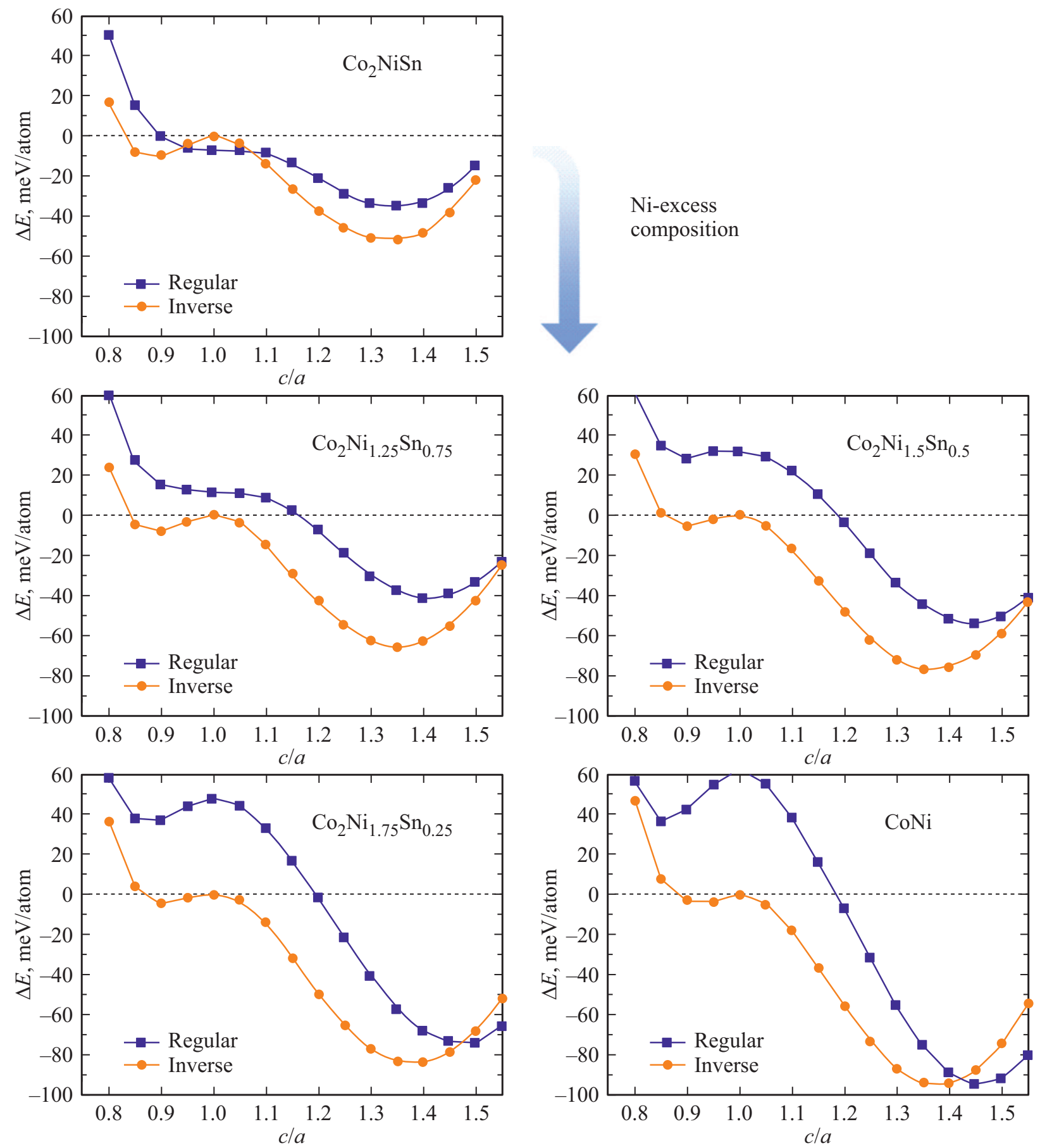

Рис. 2. Полная энергия сплавов $\mathrm{Co}_{2} \mathrm{Ni}_{1+x} \mathrm{Sn}_{1-x}(x=0,0.25,0.5,0.75,1)$ как функция степени тетрагональных искажений $c / a$ для случаев прямой и обратной гейслеровских структур. За ноль взята полная энергия сплава в обратной гейслеровской структуре при $c / a=1$.

релаксации), которая показывает, что кубическая симметрия в данных сплавах не сохраняется.

Расчеты показывают, что все исследуемые композиции обладают обратной тетрагональной структурой в мартенситной фазе, являющейся энергетически выгодной. Для всех композиций соотношение тетрагональности $c / a$ мартенситной фазы близко к 1.35 , за исключением бинарного соединения $\mathrm{CoNi}(c / a=1.4)$. Кроме того,
Ni-excess

composition 


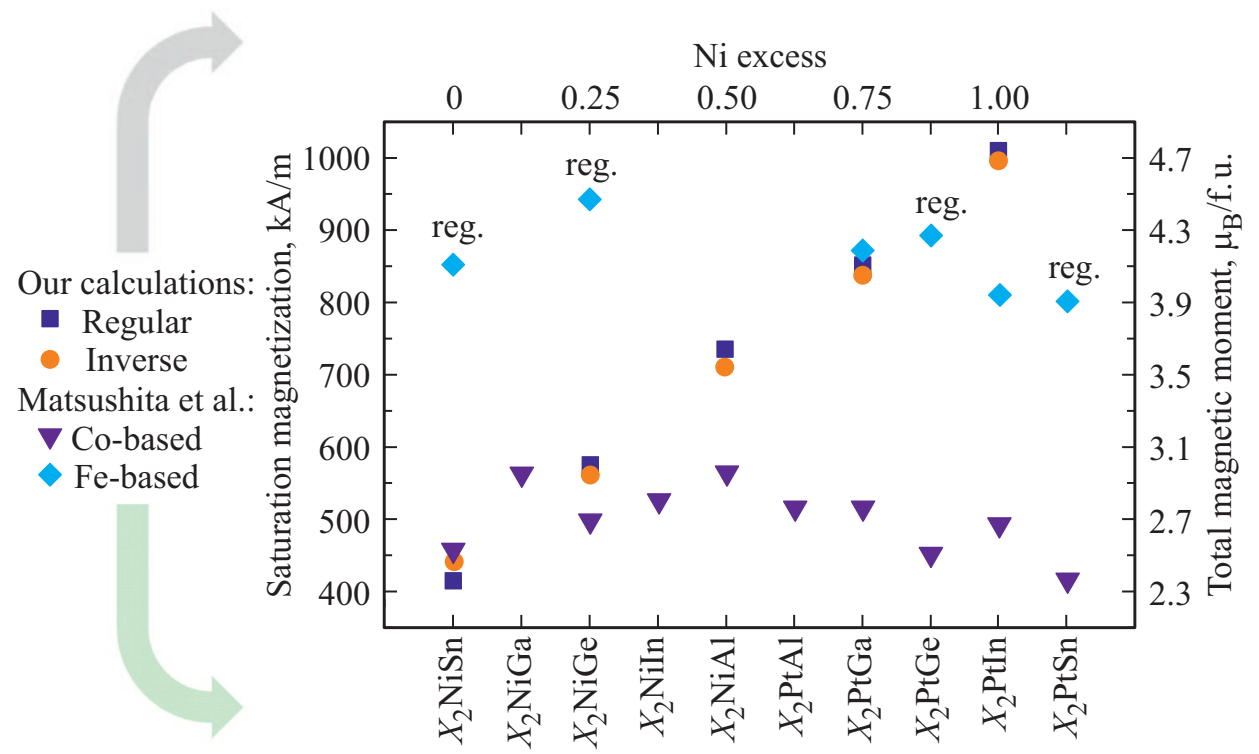

Рис. 3. Намагниченность насыщения серии сплавов $\mathrm{Co}_{2} \mathrm{Ni}_{1+x} \mathrm{Sn}_{1-x}(x=0,0.25,0.5,0.75,1)$ в сравнении с другими сплавами Гейслера на основе Со и $\mathrm{Fe}[19]$. Результаты расчетов и литературные данные приведены для мартенситных фаз в обратной гейслеровской структуре за исключением композиций на основе Fe с прямой решеткой, отмеченных на рисунке как „ґеg.“. Справа для удобства представлена шкала полных магнитных моментов сплавов $\mathrm{Co}_{2} \mathrm{Ni}_{1+x} \mathrm{Sn}_{1-x}(x=0,0.25,0.5,0.75,1)$. Для бинарного соединения $(x=1)$ величина полного магнитного момента представлена из расчета на 4 атома в формульной единице $\left(\mathrm{Co}_{2} \mathrm{Ni}_{2}\right)$.

ния мартенситной фазы является чрезвычайно важной, так как тетрагональная структура может обладать ненулевой энергией магнитокристаллической анизотропии.

Равновесные параметры для композиций с прямой и обратной гейслеровской структурой чувствительны к содержанию $\mathrm{Ni}$ и $\mathrm{Sn}$, демонстрируют тенденцию к их уменьшению при снижении концентрации Sn. Данное наблюдение может быть обусловлено бо́льшим атомным радиусом Sn по сравнению с Сo и Ni. Напротив, степень тетрагональности мартенсита для обоих типов решетки возрастает с уменьшением концентрации $\mathrm{Sn}$ (см. таблицу).

Важной характеристикой состава, претендующего на роль постоянного магнита, является высокая намагниченность насыщения $\left(M_{s}\right)$. На рис. 3 представлены результаты вычисления намагниченности насыщения для исследуемой серии $\mathrm{Co}_{2} \mathrm{Ni}_{1+x} \mathrm{Sn}_{1-x}(x=0,0.25,0.5$, $0.75,1)$, а также для сравнения приведены теоретические данные работы [19] для других сплавов Гейслера на основе Co и Fe [19]. Можно видеть, что величина $M_{s}$ для $\mathrm{Co}_{2} \mathrm{NiSn}$ согласуется с результатами теоретического исследования [19]. Авторы работы [19] показали, что намагниченность насыщения сплавов $\mathrm{Co}_{2} \mathrm{NiZ}$ и $\mathrm{Co}_{2} \mathrm{PtZ}$ лежит в пределах от 400 до $600 \mathrm{kA} / \mathrm{m}$. По сравнению с ними, сплавы на основе $\mathrm{Fe}$ обладают в два раза большей $M_{s}$, причем для некоторых из них предсказана прямая гейслеровская структура. Таким образом видно, что сильное влияние на намагниченность насыщения стехиометрических сплавов $X_{2} Y Z$ оказывает элемент $X$, тогда как замена элементов $Y$ и $Z$ сказывается незначительно. Однако из наших расчетов видно, что намагниченность насыщения можно значительно

Равновесные параметра решетки $a$ и $c$, соотношение тетрагональности $c / a$, плотность сплава $\rho$ и энергия $\mathrm{MAE}$ в $\left[\mathrm{MJ} / \mathrm{m}^{3}\right]$. Плотность сплава приведена для удобства перевода величин МАЕ из $[\mathrm{meV} / \mathrm{fu}$.$] в \left[\mathrm{MJ} / \mathrm{m}^{3}\right]$

\begin{tabular}{c|c|c|c|c|c|c|c|c|c|c}
\hline & \multicolumn{4}{|c|}{ Прямая гейслеровская структра } & \multicolumn{4}{c}{ Обратная гейслеровская структра } \\
\hline$X$ & $a, \AA$ & $c, \AA$ & $c / a$ & $\rho, \mathrm{kg} / \mathrm{m}^{3}$ & $\mathrm{MAE}, \mathrm{MJ} / \mathrm{m}^{3}$ & $a, \AA$ & $c, \AA$ & $c / a$ & $\rho, \mathrm{kg} / \mathrm{m}^{3}$ & $\mathrm{MAE}, \mathrm{MJ} / \mathrm{m}^{3}$ \\
\hline 0 & 5.36 & 7.22 & 1.34 & 9457 & -1.275 & 5.37 & 7.20 & 1.34 & 9434 & -0.106 \\
\hline 0.25 & 5.23 & 7.31 & 1.40 & 9305 & -0.404 & 5.31 & 7.08 & 1.33 & 9317 & -1.106 \\
\hline 0.50 & 5.10 & 7.36 & 1.44 & 9187 & 0.675 & 5.18 & 7.01 & 1.35 & 9362 & -1.792 \\
\hline 0.75 & 5.01 & 7.30 & 1.45 & 9080 & 1.874 & 5.11 & 7.00 & 1.37 & 9097 & -0.661 \\
\hline 1.0 & 4.92 & 7.18 & 1.46 & 8998 & 1.216 & 5.02 & 6.90 & 1.38 & 8997 & -0.396
\end{tabular}




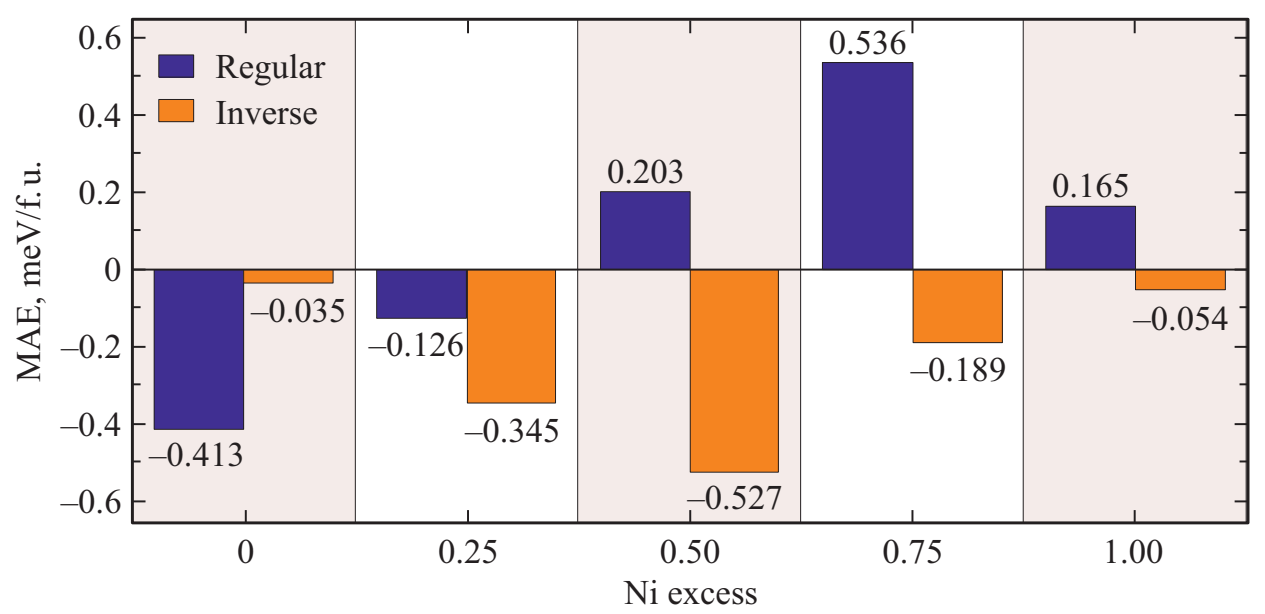

Рис. 4. Магнитокристаллическая анизотропия сплавов $\mathrm{Co}_{2} \mathrm{Ni}_{1+x} \mathrm{Sn}_{1-x}(x=0,0.25,0.5,0.75,1)$ в мартенситной фазе.

увеличитьпутем введения химического беспорядка. Так, $M_{s}$ монотонно увеличивается с ростом концентрации избыточных атомов $\mathrm{Ni} \mathrm{в} \mathrm{исследуемой} \mathrm{серии} \mathrm{композиций}$ $\mathrm{Co}-\mathrm{Ni}-\mathrm{Sn}$. В результате можно видеть, что величина намагниченности насыщения сплава $\mathrm{Co}_{2} \mathrm{Ni}_{1.75} \mathrm{Sn}_{0.25}$ практически равна $M_{s}$ сплава $\mathrm{Fe}_{2} \mathrm{PtGa}$, который будет значительно дороже в производстве из-за входящей в его состав Pt. Намагниченность бинарной композиции CoNi является наибольшей среди всех рассмотренных соединений и составляет 1010 и $998 \mathrm{kA} / \mathrm{m}$ для прямой и обратной структур, соответственно.

На рис. 4 представлены результаты вычисления магнитокристаллической анизотропии сплавов $\mathrm{Co}_{2} \mathrm{Ni}_{1+x} \mathrm{Sn}_{1-x}$ $(x=0,0.25,0.5,0.75,1)$ в мартенситной фазе. Все сплавы с обратным кристаллическим упорядочением демонстрируют отрицательные значения МАE (анизотропия типа „легкая плоскость“), причем, магнитокристаллическая анизотропия увеличивается по модулю с малой добавкой $\mathrm{Ni}$ и достигает максимального абсолютного значения $\left(-0.527 \mathrm{eV} / \mathrm{f} . \mathrm{u}\right.$. или $\left.-1.79 \mathrm{MJ} / \mathrm{m}^{3}\right)$ при $x=0.5$. Дальнейшее увеличение избытка $\mathrm{Ni}$ приводит к значительному уменьшению величины MAЕ: приблизительно в три раза для $\mathrm{Co}_{2} \mathrm{Ni}_{1.75} \mathrm{Sn}_{0.75}$ и в четыре раза $\mathrm{CoNi}$ по сравнению с композицией $x=0.5$.

В случае прямой решетки наблюдается знакопеременное поведение MAE, т.е. наблюдается смена типа анизотропии с типа „легкая плоскость“ на „легкую ось“ с увеличением содержания $\mathrm{Ni}$. Особый интерес представляют композиции $x \geq 0.5$, обладающие ненулевой положительной МАЕ и, таким образом, наиболее перспективные с точки зрения их применимости в качестве постоянных магнитов. Наибольшим значением $\mathrm{MAE}=0.536 \mathrm{meV} / \mathrm{f} . \mathrm{u} . \quad\left(1.8741 \mathrm{MJ} / \mathrm{m}^{3}\right) \quad$ обладает сплав $\mathrm{Co}_{2} \mathrm{Ni}_{1.75} \mathrm{Sn}_{0.75}$, тогда как в случае полного замещения $\mathrm{Sn}$ атомами Ni MAE уменьшается в 1.8 раза.

Для удобства сравнения в таблице представлены значения МАE в $\mathrm{MJ} / \mathrm{m}^{3}$. В целом, можно видеть, что полученные результаты для нестехиометрических составов $\mathrm{Co}_{2} \mathrm{Ni}_{1+x} \mathrm{Sn}_{1-x}$ являются близкими к значениям авторов работы [19]: $\mathrm{Co}_{2} \mathrm{NiAl}\left(-2.11 \mathrm{MJ} / \mathrm{m}^{3}\right), \mathrm{Co}_{2} \mathrm{NiGa}$ $\left(-2.38 \mathrm{MJ} / \mathrm{m}^{3}\right), \quad \mathrm{Co}_{2} \mathrm{NiIn} \quad\left(-2.22 \mathrm{MJ} / \mathrm{m}^{3}\right), \quad \mathrm{Co}_{2} \mathrm{NiGe}$ $\left(0.86 \mathrm{MJ} / \mathrm{m}^{3}\right)$ - сплавы с обратной гейслеровской тетрагональной структурой и $\mathrm{Fe}_{2} \mathrm{NiGe}\left(1.07, \mathrm{MJ} / \mathrm{m}^{3}\right)$, $\mathrm{Fe}_{2} \mathrm{NiSn}\left(1.09 \mathrm{MJ} / \mathrm{m}^{3}\right)$ - сплавы с прямой гейслеровской тетрагональной структурой.

\section{4. Заключение}

В настоящей работе с помощью методов $a b$ initio исследованы возможность структурных фазовых превращений и магнитные свойства сплавов $\mathrm{Co}-\mathrm{Ni}-\mathrm{Sn}$ с избытком $\mathrm{Ni}$. Для всех составов $\mathrm{Co}_{2} \mathrm{Ni}_{1+x} \mathrm{Sn}_{1-x}(x=0$, $0.25,0.5,0.75,1)$ показано, что они кристаллизуются в тетрагональную фазу, тогда как кубическая фаза неустойчива. Ненулевая энергия магнитокристаллической анизотропии предсказана для всех композиций как в прямой, так и в обратной геслеровских структурах. МАЕ обратной кристаллической решетки всегда отрицательна, т. е. магнитному моменту атомов Со и Ni выгодно ориентироваться вдоль плоскости. Напротив, в случае прямой решетки МАЕ демонстрирует знакопеременное поведение в зависимости от концентрации избытка $\mathrm{Ni}$, и становится положительной при $x \geq 0.5$. Положительные значения энергии магнитокристаллической анизотропии соответствуют ориентации магнитных моментов вдоль осей тетрагональной симметрии и являются важной характеристикой материала как твердотельного магнита. Таким образом, композиции $\mathrm{Co}_{2} \mathrm{Ni}_{1+x} \mathrm{Sn}_{1-x}$ при $x \geq 0.5$ заслуживают особого внимания и продолжения исследования их магнитных свойств. Стоит также отметить, что помимо химического состава большую роль играет кристаллическое упорядочение, поэтому в будущем будет интересно рассмотреть другие типы атомного порядка и исследовать их влияние на величину магнитокристаллической анизотропии. 


\section{Финансирование работы}

Исследование выполнено при финансовой поддержке РФФИ и Челябинской области в рамках научного проекта 20-42-740003.

\section{Конфликт интересов}

Авторы заявляют, что у них нет конфликта интересов.

\section{Список литературы}

[1] R. Skomski, J.M.D. Coey. Permanentmagnetism. Routledge (2019).

[2] R.W. McCallum, L.H. Lewis, R. Skomski, M.J. Kramer, I.E. Anderson. Annu. Rev. Mater. Res. 44, 1, 451 (2014).

[3] J.M.D. Coey. IEEE Trans. Magn. 47, 12, 4671 (2011).

[4] J.M.D. Coey. Scripta Mater. 67, 6, 524 (2012).

[5] M.J. Kramer, R.W. McCallum, I.A. Anderson, S. Constantinides. JOM 64, 7, 752 (2012).

[6] K.P. Skokov, O. Gutfleisch. Scripta Materialia 154, 289 (2018).

[7] J. Mohapatra, J.P. Liu. Handbook. Magn. Mater. 27, 1 (2018).

[8] A. Edström, J. Chico, A. Jakobsson, A. Bergman, J. Rusz. Phys. Rev. B 90, 1, 014402 (2014).

[9] M. Werwiński, W. Marciniak. J. Phys. D: Appl. Phys. 50, 49, 495008 (2017).

[10] L.-Y. Tian, H. Levämäki, O. Eriksson, K. Kokko, Á. Nagy, E.K. Délczeg-Czirják, L. Vitos. Sci. Rep. 9, 1, 8172 (2019).

[11] S. Bhattacharjee, S.-C. Lee. Sci. Rep. 9, 1, 8381 (2019).

[12] L. Reichel, L. Schultz, D. Pohl, S. Oswald, S. Fähler, M. Werwiński, A. Edström, E.K. Delczeg-Czirjak, J. Rusz. J. Phys.: Condens. Matter 27, 47, 476002 (2015).

[13] E.K. Delczeg-Czirjak, A. Edström, M. Werwiński, J. Rusz, N.V. Skorodumova, L. Vitos, O. Eriksson. Phys. Rev. B 89, 14, 144403 (2014).

[14] M. Werwiński, A. Edström, J. Rusz, D. Hedlund, K. Gunnarsson, P. Svedlindh, J. Cedervall, M. Sahlberg. Phys. Rev. B 98, 21, 214431 (2018).

[15] M. Werwiński, S. Kontos, K. Gunnarsson, P. Svedlindh, J. Cedervall, V. Höglin, M. Sahlberg, A. Edström, O. Eriksson, J. Rusz. Phys. Rev. B 93, 17, 174412 (2016).

[16] A. Edström, M. Werwiński, D. Iuşan, J. Rusz, O. Eriksson, K.P. Skokov, I.A. Radulov, S. Ener, M.D. Kuz'min, J. Hong, M. Fries, D.Yu. Karpenkov, O. Gutfleisch, P. Toson, J. Fidler. Phys. Rev. B 92, 17, 174413 (2015).

[17] H.C. Herper. Phys. Rev. B 98, 1, 014411 (2018).

[18] Q. Gao, I. Opahle, O. Gutfleisch, H. Zhang. Acta Mater. 186, 1, 355 (2020).

[19] Y.-I. Matsushita, G. Madjarova, J.K. Dewhurst, S. Shallcross, C. Felser, S. Sharma, E.K.U. Gross. J. Phys. D 50, 9, 095002 (2017).

[20] M. Klicpera, M. Kratochvílová, F. Malý, T. Kovaliuk, J. Valent, R.H. Colman. Intermetallics 130, 1, 107060 (2021).

[21] G. Kresse, J. Furthmüller. Phys. Rev. B. 54, 16, 11169 (1996).

[22] G. Kresse, D. Joubert. Phys. Rev. B. 59, 3, 1758 (1999).

[23] J.P. Perdew, K. Burke, M. Ernzerhof. Phys. Rev. Lett. 77, 18, 3865 (1996).

Редактор Е.В. Толстякова 